Ann. Zootech., I970, 19 (2), I43-I57.

\title{
VALEUR ALIMENTAIRE DU TOURTEAU D'OLIVE
}

\author{
M. THÉRIEZ * et G. BOULE \\ I. N. R. A. T., Ariana (Tunisie)
}

RÉSUMÉ

La distribution de Ioo g par jour de tourteau d'olive à des brebis gestantes alimentées par pâturage a réduit le gain de poids des mères (différence non significative) au cours des derniers mois de gestation et le poids des agneaux à la naissance (différence significative).

Ce tourteau a une teneur en matières azotées voisine de I $2 \mathrm{p}$. Ioo de la matière sèche et sa teneur en matières grasses peut atteindre 47 p. roo. Distribué à des béliers, il diminue la digestibilité de la ration et particulièrement celle de la fraction azotée. La digestibilité de la matière organique du tourteau dépend de son taux d'incorporation dans la ration et varie de valeurs négatives à +57 p. Ioo. La digestibilité de la matière grasse reste, par contre, très élevée (7 I à 90 p. IOO). L'activité de la flore du rumen est réduite par le tourteau d'olive, qu'il soit distribué à l'animal ou ajouté au substrat au cours de mesures in vitro. Cette action est moindre lorsque le tourteau est traité au trichloréthylène ou, mieux, hydrolysé par une solution basique à 5 p. roo. Ce traitement permet d'obtenir un résidu équivalent à un bon fourrage.

\section{INTRODUCTION}

L'extraction de l'huile d'olive laisse un résidu abondant qui représente environ 80 p. Ioo du poids des fruits traités. Deux types de traitement sont utilisés en Tunisie dans ce but. Le plus souvent les olives entières sont pressées à la presse hydraulique et le tourteau, plus communément appelé grignon d'olive, est constitué par la pellicule du fruit (épicarpe), la pulpe qui contenait l'huile (mésocarpe), la coque du noyau (endocarpe) et 1'amandon qui est la graine. Un passage au tarare permet d'éliminer une grande partie des coques et l'on obtient un grignon dénoyauté. Dans les procédés plus récents, les olives sont dénoyautées avant toute opération (type Acapulco). Le tourteau obtenu dans ce cas est constitué uniquement de la pellicule et de la pulpe du fruit, nous le désignerons ultérieurement sous le nom de pulpe d'olive pour le différencier du grignon.

Peu utilisés dans l'alimentation du bétail et d'un prix très bas, les tourteaux d'olive sont abandonnés après traitement et soumis aux intempéries. Des phéno-

* Station de Recherches sur l'Élevage des ruminants, Centre de Recherches de Clermont-Ferrand, 63-Saint-Genès Champanelle I. N. R. A. 
M. THÉRIEZ, G. BOUI,E

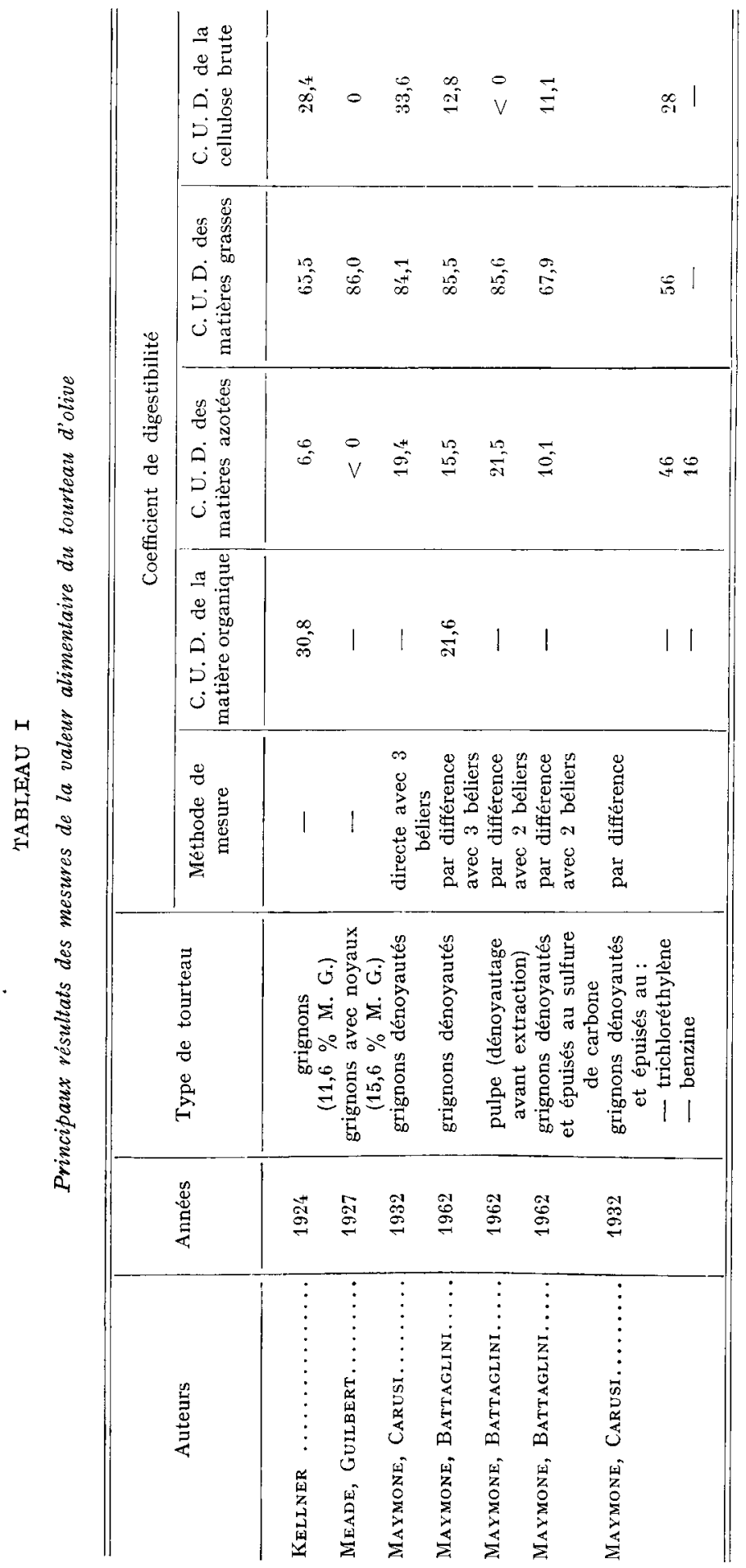


mènes de rancissement des graisses peuvent alors se produire. On doit cependant remarquer que, ni la pulpe d'olive ainsi abandonnée, ni les eaux-mères obtenues en cours d'extraction, ne fermentent.

Par contraste avec les autres tourteaux, le grignon d'olive n'est que peu utilisé dans l'alimentation du bétail. Or, les zones d'oléiculture sont souvent voisines de régions d'élevage ovin où les troupeaux souffrent régulièrement de sous-nutrition et même de famine.

Les essais menés pour déterminer soit la valeur nutritive (KELINER, I924; MAYMONE et coll., I 962, tabl. I), soit les conditions d'emploi du tourteau d'olive (CoRDIER, I947 ; BuchMAN, I963 ; CASTEJon et coll., I966), ont montré sa très faible digestibilité et, plus particulièrement, celle de sa fraction azotée. L'abondance et le faible coût de cet aliment devraient cependant rendre intéressante son utilisation dans l'alimentation des ruminants malgré sa médiocre qualité.

C'est dans le dessein de précisar les limites d'incorporation de ce tourteau et la nature des compléments alimentaires nécessaires pour sa meilleure valorisation que nous avons entrepris les différents essais rapportés ci-après. Nous avons tout d'abord déterminé sur des brebis gestantes, l'effet d'un apport de tourteau d'olive en complément du pâturage. Puis, nous avons précisé son influence sur la digestibilité de la ration globale. Dans un troisième stade, nous avons fractionné ce tourteau et nous avons mesuré l'effet des différentes fractions sur l'activité de la flore du rumen.

\section{I. - UTILISATION DU TOURTEAU D'OLIVE DANS LA RATION DES BREBIS LAITIÈRES}

Nous avons conduit un premier essai d'utilisation du tourteau d'olive sur Ioo brebis en fin de gestation. Ces animaux ont été choisis au hasard dans le troupeau de zoo laitières de race Sicilienne mis à notre disposition par I'Office de l'Enfida (roo km au Sud de Tunis).

Ce troupeau, mené selon la pratique locale, pâturait pendant la journée ; il était parqué en enclos pour la nuit. Son alimentation était basée essentiellement sur le pâturage : parcours naturels, jachères et pâturage semé de vesce-orge en automne et en hiver, chaumes de fourrages au printemps, chaumes de céréales en été. La mise bas avait lieu en février et les animaux recevaient pendant la lactation un complément de foin de vesce-orge et de céréales.

Pendant la durée de l'essai (mi-janvier à mi-février), les brebis utilisées ont été divisées en 2 lots identiques qui pâturaient ensemble pendant le jour. Le lot expérimental recevait le soir $200 \mathrm{~g}$ par tête d'un mélange moitié orge aplatie, moitié tourteau d'olive tandis que le lot témoin ne recevait rien.

TABLEAU 2

Poids moyen des agneaux à la naissance

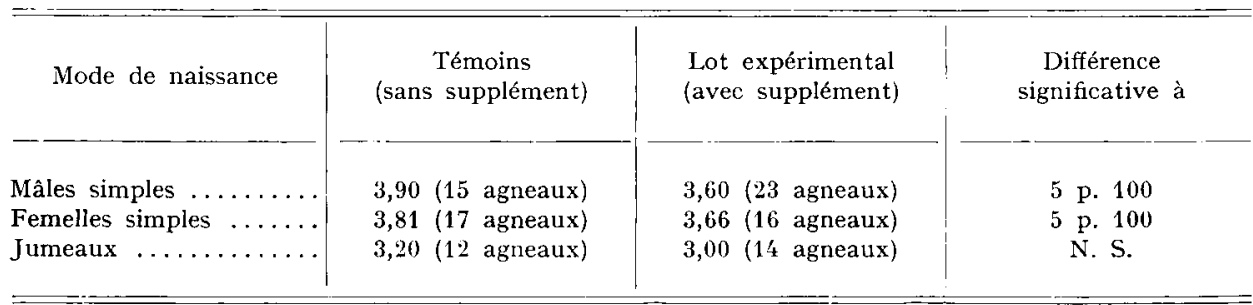


Les moyens dont nous disposions ont limité à 2 le nombre de pesées des brebis : Io bêtes de chaque lot ont été pesées au début de l'expérience puis après un mois de complémentation. Tous les agneaux ont été pesés à la naissance.

Les brebis qui recevaient le mélange complémentaire du pâturage ont eu, au cours de leur dernier mois de gestation, un gain de poids sensiblement plus faible $(5,2$ contre $6,2 \mathrm{~kg}$, différence non signi ficative), et ont mis bas des agneaux significativement plus légers que ceux du lot témoin. (tabl. 2).

Ce résultat, en accord avec ceux de CAsTEJon et al. (I966), pourrait être dû soit à une diminution de la quantité d'herbe consommée au pâturage par les brebis du fait de la complémentation, soit à une réduction de la valeur nutritive de leur ration soit à une action toxique du complément à base de tourteau d'olive.

Pour préciser lequel de ces facteurs pouvait être à l'origine des différences observées, nous avons mesuré, au laboratoire, la digestibilité de rations analogues à celles des brebis de ce troupeau.

\section{II. - COMPOSITION CHIMIQUE ET DIGESTIBILITÉ}

\section{Composition chimique}

Nous avons analysé I 2 échantillons de tourteau d'olive au cours de 4 années successives (tabl. 3).

Trois de ces échantillons étaient des grignons battus provenant de l'huilerie d'Enfida. Obtenus par pression des olives entières, ils avaient été passés au tarare pour éliminer la majeure partie des coques de noyau.

TABLEAU 3

Composition chimique du tourteau d'olive

\begin{tabular}{|c|c|c|c|c|c|c|}
\hline \multirow[b]{2}{*}{ Nature du tourteau } & \multirow{2}{*}{$\begin{array}{l}\text { Numéro de } \\
\text { l'échantillon }\end{array}$} & \multirow{2}{*}{$\begin{array}{c}\text { Teneur en } \\
\text { matière sèche }\end{array}$} & \multicolumn{4}{|c|}{ Composition par rapport à la matière sèche } \\
\hline & & & Cendres & $\begin{array}{l}\text { Matière } \\
\text { azotée }\end{array}$ & $\begin{array}{l}\text { Cellulose } \\
\text { brute }\end{array}$ & $\begin{array}{c}\text { Matière } \\
\text { grasse }\end{array}$ \\
\hline \multirow{4}{*}{ Grignons } & 1 & 79,5 & 6,0 & 8,8 & 15,5 & 30,8 \\
\hline & 2 & 88,6 & 3,4 & 10,4 & - & - \\
\hline & 3 & 76,1 & 4,2 & 8,2 & - & - \\
\hline & 4 & 84,9 & 4,3 & 10,6 & 21,9 & - \\
\hline \multirow{5}{*}{$\begin{array}{c}\text { Pulpe pression } \\
\text { ou } \\
\text { pulpe grasse }\end{array}$} & 5 & 74,5 & 4,5 & 12,2 & 26,2 & - \\
\hline & 6 & 81,0 & 6,0 & 13,5 & 19,6 & 31,9 \\
\hline & 7 & 75,0 & 3,7 & 12,9 & - & 28,8 \\
\hline & 8 & 63,0 & 4,3 & 17,9 & - & 47,7 \\
\hline & 9 & 92,5 & 4,4 & 16,1 & - & - \\
\hline \multirow{4}{*}{$\begin{array}{l}\text { Pulpe extraction } \\
\text { ou } \\
\text { pulpe dégraissée }\end{array}$} & 10 & 83,6 & 5,9 & 11,6 & 20,5 & - \\
\hline & 11 & 82,5 & 6,3 & 14,5 & 17,6 & 3,9 \\
\hline & 12 & 91,9 & $6,{ }^{\prime} 1$ & 15,8 & 229,2 & 8,8 \\
\hline & & . & & & & \\
\hline
\end{tabular}

Les 9 autres échantillons, qui provenaient d'une huilerie de la région de Tunis, avaient été obtenus par pression des fruits après dénoyautage, selon une méthode voisine du procédé "Acapulco». Ils ne contenaient qu'une très faible fraction de coques. Nous les désignerons ultérieurement sous le nom de pulpe d'olive pour les 
distinguer des grignons. 'Trois d'entre eux (pulpe extraction) avaient été traités au trichloréthylène pour en extraire 1'huile alors que les 6 autres n'avaient subi qu'une pression (pulpe pression).

Les grignons ont une teneur en matières azotées voisine de $9 \mathrm{p}$. Ioo, inférieure à celle de la pulpe (I4 p. roo). Cette différence est due à une proportion plus importante de coques qui représentent environ la moitié de la masse du grignon, leur teneur en matière azotée est voisine de 2 p. Ioo.

La teneur en matière grasse des tourteaux obtenus par pression (grignon et pulpe) est très élevée, elle dépasse en général $30 \mathrm{p}$. Ioo de la matière sèche ; le traitement au trichloréthylène la réduit à 6 p. Ioo. La teneur en cellulose brute est également élevée ; elle est voisine de 20 p. IoJ.

\section{Digestibilité}

Nous avons mesuré le coefficient de digestibilité de 5 des I2 échantillons analysés (I échantillon de grignons, $I$ de pulpe extraction et 3 de pulpe pression).

Ces tourteaux étant peu appétibles lorsqu'ils sont distribués seuls, nous avons đû les mélanger à de l'orge. Le mélange complémentait une ration de fourrages secs (paille de blé ou foin de vesce-avoine).

Deux premiers essais (échantillons I et 4) nous ayant montré que l'addition de pulpe d'olive modifiait la digestibilité propre de la ration, nous avons ultérieurement, fait varier la quantité de tourteaux distribuée en maintenant fixes les apports de fourrage et d'orge (essais avec les échantillons 5 et ro) tandis que, pour l'échantillon 6 , c'est la quantité de mélange $5^{\circ} \mathrm{p}$. Ioo orge-50 p. Ioo tourteau qui variait. Pour l'ensemble des mesures, la proportion de tourteau d'olive a varié de $8, \mathrm{I}$ à 24,4 p. Ioo.

Au cours de chacun des essais, nous avons mesuré successivement et avec les mêmes animaux la digestibilité du fourrage seul, celle de la ration fourrage + orge puis celles des rations complètes fourrage + mélange orge et tourteau d'olive. Les périodes de mesure du CUD duraient 7 jours et nous utilisions 4 béliers par lot. Le CUD du tourteau d'olive a été estimé par différence en supposant qu'il n'y avait pas de phénomène de digestibilité associative. Cette méthode de calcul rend aléatoire les résultats obtenus lorsque le tourteau d'olive représente moins de ro p. roo du régime.

Dans sept mesures sur huit, le coefficient de digestibilité de la ration complète (fourrage + orge + tourteau d'olive), a été plus faible que celle de la ration fourrage + orge (tabl. 4). Cette différence, peu importante (inférieure à 2 points) avec la pulpe traitée au trichloréthylène peut atteindre $I_{3}$ points avec la pulpe grasse. La chute de la digestibilité, maximum lorsque le tourteau d'olive représente Io $\mathrm{p}$. Ioo environ de la ration, est moins marquée lorsque le pourcentage de tourteau est supérieur à Io p. Ioo (échantillons 5, 6 et Io distribués selon 2 niveaux différents) (tabl. 4).

En outre, l'addition de tourteau d'olive accroît les écarts de digestibilité observés d'un animal à l'autre. L'écart type des 4 résultats d'un même lot, qui est compris entre 0,9 et 2 , I unités pour des rations de fourrage + orge, est presque doublé $(\mathbf{I}, 3$ à 4,I unités) lorsqu'on ajoute du tourteau d'olive.

L'effet de l'apport de tourteau d'olive dépend enfin de la qualité de la ration initiale ; il est d'autant plus important que celle-ci était moins digestible.

L'addition de pulpe ou de grignon d'olive entraîne d'autre part une diminution de la digestibilité des matières azotées de la ration (fig. I). Le taux de matières azotées 


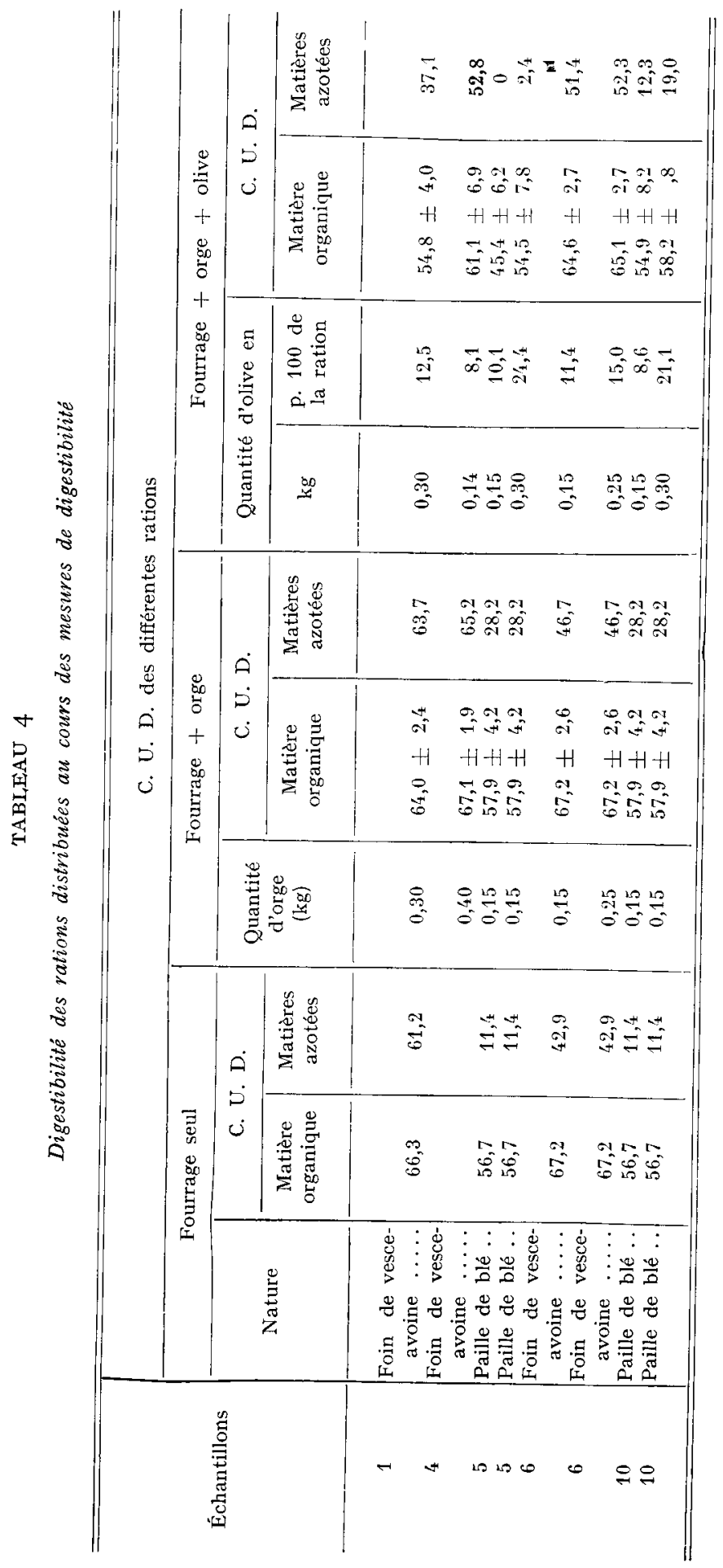


digestibles observé est de 0,4 à 3,3 unités inférieur à celui que 1'on obtient par le calcul en utilisant la relation générale entre teneur en matière azotée digestible $(\mathrm{Y})$ et teneur en matière azotée totale $(\mathrm{X})$ que nous avons établie, par ailleurs, pour les aliments locaux $(\mathrm{Y}=0,94 \mathrm{IX}-3,45)$.

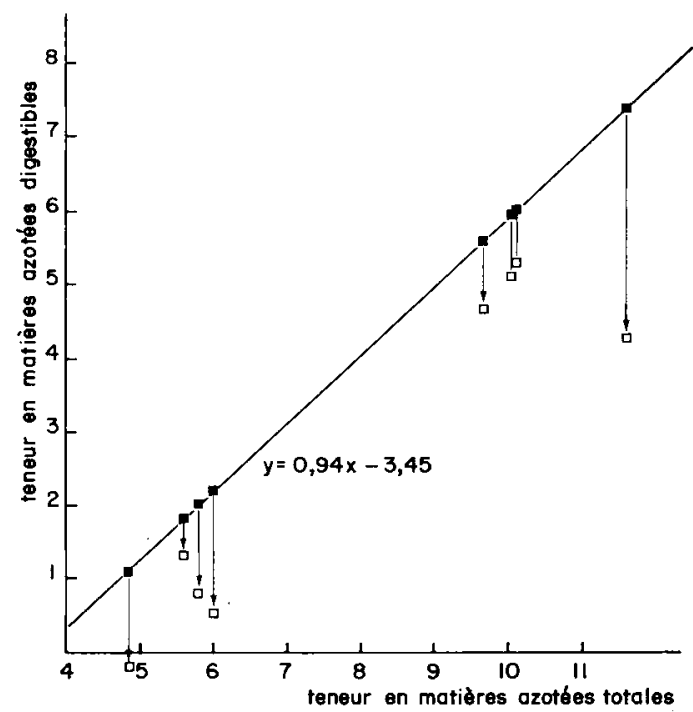

FIc. I. - Influence du tourteau d'olive sur la digestibilité des matières azotées de la ration

- : Valeurs théoriques calculées

$\square$ : Valeurs réclles observées

I a digestibilité des matières grasses des différentes rations reste, par contre, très élevée, que l'on distribue un tourteau traité ou non au trichloréthylène.

Si on calcule la digestibilité des tourteaux d'olive, en admettant qu'ils n'ont aucun effet sur la digestibilité de la ration (foin + orge), les résultats obtenus, tant pour la matière organique que pour les matières azotées sont très variables (tabl. 5 ) ;

TABIEAU 5

Digestibilité du tourteau d'olive

\begin{tabular}{|c|c|c|c|c|c|c|}
\hline \multirow{2}{*}{ No échantillon } & \multirow{2}{*}{$\begin{array}{l}\text { Pourcentage } \\
\text { de tourteau } \\
\text { dans la ration }\end{array}$} & \multicolumn{2}{|c|}{$\begin{array}{l}\text { Teneur de la ration en } \\
\text { p. } 100 \text { de la matiere sèche }\end{array}$} & \multicolumn{3}{|c|}{ C. U. D. de l'olive } \\
\hline & & $\begin{array}{c}\text { Matières } \\
\text { grasses }\end{array}$ & $\begin{array}{c}\text { Matières } \\
\text { azotées }\end{array}$ & $\begin{array}{c}\text { Matière } \\
\text { organique }\end{array}$ & $\begin{array}{c}\text { Matières } \\
\text { azotées }\end{array}$ & $\begin{array}{c}\text { Matières } \\
\text { grasses }\end{array}$ \\
\hline 1 & 12,5 & $5, \overline{5}$ & 11,5 & 45,7 & 23,6 & 71,2 \\
\hline 4 & 8,1 & 4,6 & 10,1 & $<0$ & $<0$ & - \\
\hline 5 & 10,1 & 6,0 & 4,9 & $<0$ & $<0$ & 一 \\
\hline 5 & 24,4 & 9,6 & 5,7 & 43,7 & 13,4 & - \\
\hline 6 & 11,4 & 4,7 & 9,6 & 47,6 & 76,5 & 89,3 \\
\hline 6 & 15,0 & 6,7 & 10,0 & $57, \mathbf{6}$ & 66,8 & 90,0 \\
\hline 10 & 8,6 & $:, 5$ & 5,6 & 18,9 & $<0$ & - \\
\hline 10 & 21,1 & 3,0 & 6,0 & 69,4 & 28,0 & 一 \\
\hline
\end{tabular}


ils sont d'autant plus élevés que le taux d'incorporation du tourteau dans la ration est plus fort (échantillons 5, 6 et ro).

Cette variation du CUD avec le taux d'incorporation permet d'expliquer l'hétérogénéité des résultats obtenus par différents auteurs et que rapporte NEHRING (I965). La digestibilité des matières azotées du grignon non épuisé en particulier, varie, selon les auteurs, de valeurs négatives (MEADE, et GUILBERT, I927) à + 6,6 p. IOO selon KELINER et +20 p. IOO selon MAYMONE et al. (I962), tandis qu'elle atteint 46 p. Ioo, selon ces mêmes auteurs, pour le même tourteau épuisé au trichloréthylène.

Nous avons également observé cette action du trichloréthylène (échantillons 5 et Io) sur un lot d'olives dont une partie avait été épuisée après pression. Ce traitement complémentaire a permis d'augmenter le CUI de la matière organique de 43,7 à 69,4 p. Ioo et celui des matières azotées de 13,4 à 28,0 p. Ioo.

La variation du CUD des tourteaux d'olive selon le taux d'incorporation dans la ration peut être due à plusieurs facteurs dont la méthode de travail (tourteau en complément d'une ration) et le mode de calcul qui conduisent à reporter sur les tourteaux les erreurs de détermination du CUD de la ration initiale. Il en résulte une erreur relative d'autant plus importante que le tourteau représente une fraction plus faible de la ration.

D'ailleurs le tourteau d'olive diminue la digestibilité propre de la ration initiale. En effet, la teneur en matières azotées digestibles observée sur les 6 rations contenant du tourteau pression est encore inférieure à 0,6 points (différence non significative) à celle que l'on obtient par calcul en estimant que les matières azotées de l'olive sont totalement indigestibles. I1 n'est donc pas possible de déterminer avec précision le CUD du tourteau d'olive, d'une part à cause de sa faible appétibilité, qui limite à 25 p. Ioo le taux d'incorporation et, d'autre part, du fait de son action sur la digestibilité de la ration initiale. Les valeurs réelles doivent être voisines des valeurs observées lorsque la proportion de tourteat1 est la plus élevée car son effet dépressif sur la ration et l'erreur systématique sont alors les plus faibles.

Ces valeurs seraient de $60 \mathrm{p}$. xoo pour le CUD de la matière sèche du tourteau pression et de $70 \mathrm{p}$. Ioo pour celui du tourteau extraction.

\section{III. - ACTION DU TOUR'TEAU D'OLIVE SUR I'ACTIVITÉ DE LA FLORE DU RUMEN}

L'effet dépressif du tourteau d'olive sur le CUD de la ration pouvant être dù à une modification de l'activité de la flore du rumen, nous avons cherché à mettre une telle action en évidence.

Nous avons d'abord déterminé l'action du tourteau d'olive chez l'animal qui en consomme, en comparant le dégagement gazeux et la digestion in vitro d'un foin de référence, par des jus de rumen prélevés, soit sur des béliers qui recevaient du tourteau d'olive, soit sur des témoins qui n'en recevaient pas.

Nous avons ensuite mesuré l'action in vitro du tourteau d'olive en l'ajoutant au substrat dans les mesures de dégagement gazeux, au foin de référence, dans les déterminations du CUD in vitro. 
En outre, après épuisement du tourteau par différents solvants et fractionnement par hydrolyse, nous avons mesuré l'action de chacun des résidus ou fractions obtenus sur le CUD in vitro de ce même foin.

\section{Matériel et méthodes}

Ces deux types de mesures ont été faits avec des échantillons de jus de rumen prélevés sur des béliers munis de fistules. Ces animaux, maintenus en bergerie, recevaient du foin de vesceavoine ad libitum et Ioo $\mathrm{g}$ d'orge aplatie. Lorsqu'on voulait tester l'effet du tourteau d'olive sur l'activité de la flore du rumen, les animaux donneurs recevaient en outre too g par jour de "pulpe pression " riche en matière grasse.

Les échantillons de jus de rumen, qui provenaient toujours de plusieurs béliers ( 2 à 4 ), étaient prélevés $\mathrm{I} h \mathrm{~h} 3 \mathrm{à} 2$ heures après le repas. Les animaux donneurs consommaient le même type de régime depuis 2 semaines au minimum. Les prélèvements étaient recueillis dans un même récipient, mélangés, filtrés sur une double épaisseur de gaze chirurgicale et dilués au $\mathrm{r} / 5$ dans une solution de salive artificielle saturée au $\mathrm{CO}_{2}$ (MAC Dougall, 1948). Toutes ces opérations et les mesures ultérieures étaient effectuées au bain-marie à $3^{\circ} \mathrm{C}$.

Nous avons utilisé tout au long de chacune des séries de mesures du dégagement gazeux et du CUD in vitro, les mêmes substrats : mélange $2 / 3$ orge et $I / 3$ de foin de luzerne pour les premières, foin de vesce-avoine pour les secondes. Ces aliments, comme les échantillons de tourteau d'olive auxquels nous les avons mélangés dans certaines mesures, avaient été broyés dans un broyeur à marteau équipé d'une grille à mailles de $0,5 \mathrm{~mm}$.

Les mesures de dégagement gazeux ont été réalisées dans des cellules de Warburg où $2 \mathrm{ml} \mathrm{de}$ jus de rumen dilué étaient mélangés à un volume égal d'eau dans lequel le substrat était en suspension à raison de Io g par litre. Les mesures effectuées à l'obscurité duraient deux heures.

Les mesures de digestibilité in vitro ont été faites d'après la méthode de Tillexy et TerRY (1963). Nous nous sommes limités à la digestion microbienne en mélangeant $50 \mathrm{ml}$ de jus de rumen dilué et $600 \mathrm{mg}$ d'aliment à digérer $(600 \mathrm{mg}$ de foin de vesce-avoine seul dans les tubes témoins, $400 \mathrm{mg}$ de foin et $200 \mathrm{mg}$ de produit à tester dans les autres). Les variations de $\mathrm{pH}$ ont été limitées par l'addition de $2 \mathrm{ml}$ de solution normale de carbonate de sodium après 6 heures et 24 heures de mesures.

\section{Résultats et discussion}

L'activité du jus de rumen des animaux qui reçoivent du tourteau d'olive est plus faible que celle des jus provenant des témoins. Au cours de 3 essais successifs, la consommation du tourteau d'olive par l'animal s'est traduite par une réduction moyenne de $40 \mathrm{p}$. Ioo du dégagement gazeux et de I 2 p. Ioo (différence significative entre I et $5 \mathrm{p}$. I0o) du CUD in vitro du foin de référence (tabl. 6).

Le mélange, dans les cellules de Warburg, de tourteau d'olive au substrat de référence orge-luzerne a un effet analogue qui est d'autant plus important que la proportion d'olive est plus élevée. Le dégagement gazeux mesuré lorsque le tourteau d'olive constitue la totalité du substrat ne représente plus que $5^{\circ} \mathrm{p}$. Ioo de celui que l'on observe avec le substrat de référence. Cette réduction se produit de façon analogue, que le jus de rumen provienne d'animaux nourris au foin ou qui reçoivent Io p. Ioo d'olive dans leurs rations. L'effet d'un apport de tourteau dans la ration est plus important que celui de l'addition du même tourteau dans les cellules de mesure (fig. 2).

Cette différence pouvant être due à la durée insuffisante de nos mesures ( 3 heures dans les cellules de Warburg), nous avons répété ce schéma expérimental lors des déterminations du CUD in vitro pendant 48 heures. Dans ce cas, l'effet observé est l'inverse de celui obtenu au Warburg. L'apport du tourteau d'olive dans les tubes de réaction a un effet plus important sur le CUD du foin que celui de ce même tourteau par l'animal. Les résultats obtenus en présence de pulpe d'olive sont les mêmes quelle que soit l'origine du jus de rumen (tabl. 6). 
TABLEAU 6

Influence du tourteau d'olive sur l'activité de la fore du rumen

\begin{tabular}{|c|c|c|}
\hline \multirow{2}{*}{ Régime de l'animal donneur } & \multicolumn{2}{|c|}{$\begin{array}{l}\text { Variation de pression dans les cellules de Warburg } \\
\qquad(\mathrm{mm})\end{array}$} \\
\hline & $\begin{array}{l}\text { Substrat orge luzerne } \\
\qquad 100 \text { p. } 100\end{array}$ & $\begin{array}{l}\text { Substrat orge-luzerne } 75 \text { p. } 100 \\
\quad+\text { olive } 25 \text { p. } 100\end{array}$ \\
\hline Foin de vesce-avoine $!$ orge & $71 \mathrm{~mm}(100)$ & $5.3 \mathrm{~mm}(75)$ \\
\hline $\begin{array}{c}\text { Foin de vesce-avoine }+ \text { orge } \\
+ \text { tourteau d'olive }\end{array}$ & $43 \mathrm{~mm}(60)$ & $3 \prime \operatorname{tnm}(18)$ \\
\hline \multirow{4}{*}{ Foin de vesce-avoine + orge } & \multicolumn{2}{|c|}{ I)igestibilité in vitro en p. 100 de la matière sèche } \\
\hline & lioin de vesce-avoine & 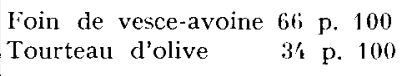 \\
\hline & \multicolumn{2}{|c|}{$\begin{array}{l}\text { (Différence S) } \\
\quad p<0,01\end{array}$} \\
\hline & $4^{\prime}, 2(100)$ & $33,2(75)$ \\
\hline $\begin{array}{c}\text { Foin de vesce-avoine }+ \text { orge } \\
+ \text { olive }\end{array}$ & $\begin{array}{c}\text { (Différence S) } \\
0,05<p<0,01 \\
38,8(88) \\
\\
\end{array}$ & $\begin{array}{l} \\
\\
\\
\\
\text { ence S) } \\
b<0,0 \mathrm{~L}\end{array}$ \\
\hline
\end{tabular}

Résultats exprimés en valeurs absolues et en valeurs relatives par rapport au témoin.

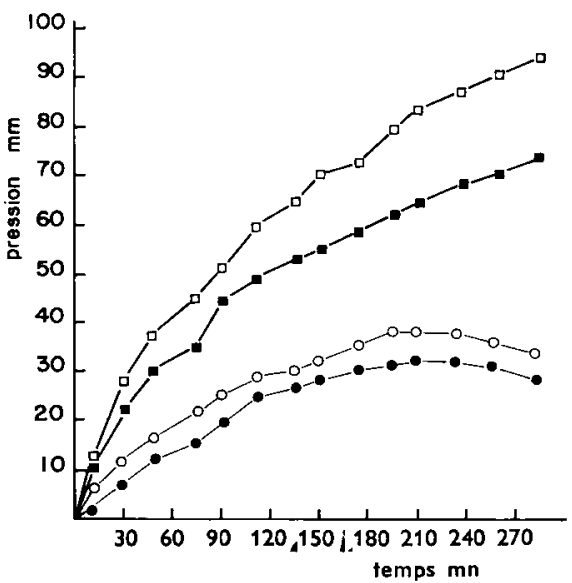

FIG. 2. - Influence du tourteau d'olive sur l'activité de la flore du rumen: variation de pression dans les cellules de Warburg

-

- - et 0 - 0 : ro p. roo de tourteau dans la ration du bélier

$\square-\square$ et $0-0$ : pas de tourteau dans le substrat

- - et - - : 25 p. roo de tourteau dans le substrat 
On peut donc penser que le tourteau d'olive contient une ou plusieurs substances qui, solubilisées ou hydrolysées lentement en présence du jus de rumen entraînent une réduction de son activité et pourraient réduire la digestibilité de la ration.

Pour préciser quelques propriétés de ces substances, nous avons traité un échantillon de pulpe grasse soit par extraction, soit par fractionnement, et nous avons mesuré l'effet de l'addition des résidus du traitement sur le CUD in vitro du foin de vesce-avoine de référence.

Nous avions pu déduire des mesures de digestibilité in vivo, que le facteur recherché est soluble dans le trichloréthylène; il l'est également, mais à un moindre degré, dans le tétrachlorure de carbone. En effet, l'addition de pulpe d'olive traitée par ce solvant a un effet dépressif moindre que celui d'une même quantité de pulpe grasse. Si l'on prend pour base roo le CUD in vitro du foin, lorsqu'on ajoute de la pulpe d'olive, on obtient pour le mélange une valeur de 80 avec la pulpe grasse et de 85 avec la pulpe dégraissée au tétrachlorure de carbone (différence non significative).

L'hydrolyse alcaline $(\mathrm{NaOH}$ ou $\mathrm{KOH}$ à $5 \mathrm{p}$. 100 pendant 30 minutes à 1'ébullition) permet, par contre, d'obtenir un résidu dont la digestibilité in vitro est supérieure à celle du foin de référence. Si la digestibilité prise pour base a la valeur ıoo, celle des mélanges foin + pulpe grasse et foin + résidu solide de l'hydrolyse a pour valeur 85 et 107 (différence hautement significative).

Après filtration du résidu solide du tourteau, l'acidifaction à $\mathrm{pH} 2$ du filtrat permet d'obtenir un précipité qui contient environ 20 p. Ioo de matières azotées et représente le quart de la masse de pulpe traitée. Remis en solution à $\mathrm{pH} 7$, il réduit 1a digestibilité $i n$ vitro du foin de référence dans les mêmes proportions que la pulpe d'olive et c'est la seule fraction du tourteau qui manifeste cet effet (tabl. 7).

\section{TABLEAU 7}

Influence de l'addition de pulpe d'olive ou de ses différentes fractions sur la digestibilité in vitro d'un foin de vesce-avoine

(Résultats exprimés en p. Ioo de la digestibilité du foin seul)

\begin{tabular}{|c|c|c|c|c|c|}
\hline & Foin seul & $\begin{array}{c}\text { Foin }+ \\
\text { Pulpe d'olive }\end{array}$ & $\begin{array}{l}\text { Foin + résidu } \\
\text { de l'hydrolyse } \\
\text { alcaline }\end{array}$ & $\begin{array}{l}\text { Foin + Précipité } \\
\text { acide de la } \\
\text { fraction liquide }\end{array}$ & $\begin{array}{l}\text { Foin t fraction } \\
\text { liquide résiduelle }\end{array}$ \\
\hline C. U.D. . . . . & 100 & 84,7 & 106,7 & 85,5 & 120 \\
\hline
\end{tabular}

\section{DISCUSSION ET CONCLUSION}

L'utilisation du tourteau d'olive sur brebis gestantes a eu un effet opposé à celui que nous espérions : les agneaux issus des mères complémentées étaient plus légers que les témoins. Plusieurs causes peuvent être envisagées pour expliquer ce moindre poids, tout d'abord une action directe sur les constituants de la ration dont la digestibilité serait réduite, mais également un effet inhibiteur sur 1'activité de la flore du rumen et un effet toxique sur l'animal qui consomme le tourteau d'olive. 
La diminution de la digestibilité peut être due tout d'abord à un excès de matières grasses dans la ration : le tourteau d'olive " pression " serait moins digestible que le tourteau "extraction " car il contient plus d'huile. Cette action des lipides sur la digestibilité chez les Ruminants a été mise en évidence par ERWIN et al. (I956) qui ont montré que l'apport de $7 \mathrm{p}$. Ioo de suif diminuait la digestibilité de la cellulose brute et de la matière sèche de la ration chez le Bœuf. De même BuYSSE (I962) a montré sur le mouton que, si un apport de I5 ou $45 \mathrm{~g}$ de suif (qui augmentait la teneur en matières grasses du régime jusqu'à $6 \mathrm{p}$. I0o) n'avait pas d'effet sur la digestibilité, l'addition de 60 à $90 \mathrm{~g}$ du même suif diminuait le CUD de la matière organique, de la matière azotée et de la cellulose brute. HALE et al. (I965) n'observent, par contre, aucun effet lorsqu'ils ajoutent $4 \mathrm{p}$. Ioo de suif à une ration pour le bouf tandis que, selon Splin et al. (I963) un apport de 4 p. Ioo de suif ou d'huile hydrolysée augmenterait la digestibilité de la matière sèche, de la matière azotée et de la cellulose brute. Si les Ruminants sont très sensibles à un apport de graisse dans la ration, les résultats précédents montrent cependant que l'effet n'est important que lorsque l'apport de matière grasse dépasse 5 p. Ioo (VANSchoubroEK, I966). Or, dans nos différentes mesures de digestibilité, nous n'avons dépassé ce seuil de 5 p. Ioo de matière grasse que dans un seul cas (échantillon $5 b: 8$, I p. Ioo de matières grasses d'olive). Si la matière grasse a réduit la digestibilité de la ration, son effet a dû être limité et ne saurait en aucun cas atteindre les valeurs observées.

La matière grasse peut, par contre, être à l'origine de la réduction d'activité du jus de rumen mesurée au Warburg. En effet, comme l'ont montré CzERKAwsKI et al. (1965), un apport de $90 \mathrm{~g}$ par 24 heures d'un mélange d'acides gras en $C_{16}$ et $C_{18}$ entraîne une réduction d'environ $5^{\circ} \mathrm{p}$. Ioo du volume de méthane dégagé sans toutefois modifier la digestion de la cellulose. Or, dans nos essais, les béliers donneurs consommaient Ioo g de tourteau pression par jour soit environ $30 \mathrm{~g}$ d'huile. Dans ce cas comme dans le précédent, si les matières grasses ont eu un effet, les quantités consommées sont restées limitées et ne peuvent donc suffire à expliquer la totalité du phénomène observé.

I1 doit donc exister dans l'olive d'autres facteurs qui réduisent la digestibilité de la ration et sont solubles dans certains solvants des corps gras. Le trichloréthylène qui, comme le tétrachlorure de carbone, est utilisé pour extraire les matières grasses, permettrait d'éliminer également ces substances à la différence du tétrachlorure dont l'effet est peu important.

La présence de tels facteurs a été mise en évidence dans certains aliments. Ainsi CHARLET-LÉRY et al. (I955) ont observé sur porcs et moutons une réduction de la digestibilité de la fraction azotée du régime en présence de marc de pomme et ils attribuent cette diminution à une inhibition enzymatique exercée par les tannins au niveau du tube digestif. Ces substances agiraient également sur les matières azotées qu'elles tanneraient, réduisant ainsi leur solubilité et leur digestibilité. Or, comme l'a observé ZELTER (I968), les matières azotées du tourteau d'olive sont très peu solubles : moins de I,5 p. Ioo de l'azote total de la pulpe grasse est soluble dans une solution de pepsine ou de trypsine. Le traitement au trichloréthylène, qui améliore la digestibilité de la matière organique et des matières azotées du tourteau, augmente jusqu'à 7,I p. Ioo la solubilité de ces dernières. Selon VAsquEz-RONCERo (I965), on trouve dans l'olive des tannins pyrogalliques qui représentent 0,3 p. Ioo de la matière sèche, tandis que selon PEYrot (I936) cette teneur s'élèverait jusqu'à 4 p. Ioo. Cette 
réduction de la solubilité des matières azotées pourrait être à l'origine des écarts que nous avons observés entre les teneurs en matières azotées digestibles déterminées par la mesure de digestibilité in vivo et estimées par le calcul.

Les tannins, ou d'autres substances, peuvent également avoir une action sur la digestibilité de la ration en réduisant l'activité de la flore du rumen. Cette action inhibitrice de l'olive sur des microorganismes a été mise en évidence par FLEMMING et ETCHELIS (I967) qui étudient le développement des fermentations lactiques se produisant lors de la préparation des olives vertes pour la consommation. Selon ces auteurs, un composé phénolique simple pourrait être l'agent inhibiteur de ces fermentations. De tels composés existent dans l'olive comme l'a montré CANZONERI (I964) qui a isolé d'une huile d'olive très amère eugenol, cathéchine et pyrocathéchine. Or, nouts avons utilisé pour nos mesures des tourteaux très riches en matière grasse (30 p. I0o de la matière sèche en moyenne). Un traitement au trychloréthylène extrait ces huiles de moindre qualité et l'amélioration de la digestibilité du tourteau qui en résulte peut être due à l'élimination de ces substances plutôt qu'à celle de la matière grasse qui est très digestible, malgré la dégradation qu'elle subit au cours de la conservation. Le rancissement de l'huile dans les tourteaux ne semble pas être la cause de la chute de digestibilité observée in vitro puisque nous avons obtenu les mêmes résultats avec la pulpe d'olive conservée plus d'un an en tas et de la pulpe fraîche prélevée en cours de traitement dans le pressoir.

Deux types de substances tannoïdes peuvent donc être à l'origine des effets observés, d'une part des composés simples qui inhiberaient les fermentations et d'autre part des tannins qui insolubiliseraient les matières azotées de la ration. Cela expliquerait la diminution de la valeur nutritive du régime que nous avons observée.

Ces deux types d'action qui entraînent une diminution du niveau alimentaire de la mère semblent cependant insuffisants pour réduire le poids des agneaux. Ce n'est en effet qu'à la suite d'une sous-alimentation sévère que l'on peut réduire le poids d'un agneau à la naissance. Or, les gains des brebis au cours du dernier mois de gestation $(5,2$ et $6,2 \mathrm{~kg})$ montrent que le régime qu'elles recevaient était satisfaisant. Le tourteau d'olive distribué a donc eu, outre l'effet dépressif sur la valeur alimentaire de la ration, un effet toxique sur les agneaux in utero. Nous ignorons la nature du facteur en cause et son mode d'action.

Le tourteau d'olive qui est actuellement inemployé ne peut donc être utilisé dans l'alimentation du bétail, tel qu'il est produit par les huileries. Le traitement au trichloréthylène, destiné à a méliorer le taux d'extraction de l'huile, permet de réduire les effets nocifs du tourteau, dont la digestibilité augmente de Io p. Ioo environ, soit par augmentation de la solubilité des matières azotées soit par élimination de substances inhibitrices de la flore du rumen.

L'emploi du tourteau d'olive comme constituant courant des rations de ruminants suppose en outre un traitement supplémentaire (hydrolyse alcaline) destiné à éliminer le facteur inhibiteur de la flore du rumen. L'aliment obtenu, dégraissé et appauvri en matières azotées, a la digestibilité d'un bon fourrage sec et son emploi peut être envisagé comme tel. L'effet toxique que nous avons observé sur brebis gestante limite cependant la valeur alimentaire du tourteau et restreint son emploi aux bêtes à l'entretien ou en période de disette.

Un développement ultérieur de ce travail devrait permettre de répondre aux hypothèses émises sur la nature du ou des facteurs en cause et par là de préciser la 
nature du traitement à faire subir au tourteau. L'utilisation dans la pratique courante de ce sous-produit permettrait non seulement de valoriser la production oléicole mais surtout de disposer, en Tunisie, de 200 ooo tonnes de pulpe d'olive pression soit Ioo à 120 ooo tonnes de pulpe extraction ou, sur la base de $0,45 \mathrm{UF} / \mathrm{kg}$, de 50 millions d'unités fourragères. Or, on estime (LE HOUERou et FroMENT, I966) que la production fourragère annuelle de la Tunisie, toutes ressources comprises, est voisine de 500 millions d'unités fourragères. Nous disposerions donc d'un moyen d'accroître rapidement les ressources fourragères dans une partie du pays (zones Centre et Sud) qui en est démunie.

Reçu pour publication en avril 1970.

\section{SUMMARY}

\section{FEEDING VALUE OF OLIVE CAKES}

Ioo ewes were distributed randomly at the end of gestation in two groups at pasture on seedlings of vetch and oats. One of these groups received a supplement containing Ioo $\mathrm{g}$ barley and $100 \mathrm{~g}$ olive cakes. The intake of this supplement diminished weight gain of the ewes $(5,2 \mathrm{~kg}$ for the supplemented group, 6,2 for the control group) and birth weight of the lambs $(3,6 \mathrm{~kg}$ compared with $3.9 \mathrm{~kg}$ for non-sib males $(\mathbf{P}<0.05$ ) (table 2 ).

2. The olive cakes were analysed and their digestibility measured on rams. They contained I 3 p. I oo total nitrogenous matter, $20 \mathrm{p}$. Ioo crude fiber and $30 \mathrm{p}$. I oo fat following the samples obtained by pressing (table 3 ). The digestibility of the organic matter and of protein varied according to their level of incorporation into the ration, from negative values until $+57 \mathrm{p}$. Ioo for the former and $+76 \mathrm{p}$. Ioo for the latter. The digestibility of fat was high (7I.2 to 90.0 p. I oo) (table 5). When olive cakes were added to the ration, the digestibility of the organic matter and of protein decreased.

3. This reduction of digestibility may be due to a decrease of the activity of the bacterial flora as shown by the in vitro measure of gas production and of the D. U. C. The latter decreased ( 40 to $50 \mathrm{p}$. 100 ) when the animals were fed olive cakes or when this oil-cake was added to the substrate submitted to fermentation.

4. Trichlorethylene extraction and alkaline hydrolysis reduced this negative effect upon digestibility. When applying the second treatement, one may obtain an oil-cake of the same value as a good forage.

The nature of the subtances responsible for these effects and the conditions of utilization of this oil-cake are discussed in this paper.

\section{RÉFÉRENCES BIBLIOGRAPHIQUES}

BUchmand E., I963. Compte rendu d'une expérience d'alimentation comparative du bétail avec des pulpes et des feuilles d'oliviers. 2e conférence internationale des techniciens oléicoles. TE 25, I-5.

Buysse F., 1962. Influence de quantités croissantes de matière grasse sur la digestibilité des éléments, nutritifs, en particulier de la cellulose brute chez les ruminants (moutons). Rev. A gricult. Brussels.. 15, 343-352.

Canzoneri F., I964. Grasas y aceites., 15, 87-97.

Castejon F., Perez-Cuesta M., 1966. El orujo de aceituna en lo alimentacion de los ovinos. Congrès mondial d'alimentation, Tome II, 399-4I3.

Charlet-I.tery G., Leroy A.-M., Zelter S.-Z., I955. Recherches sur l'efficacité alimentaire des marcs de pommes fermiers. $V$. Étude chez le mouton et le porc de la digestibilité apparente des constituants de marc de pommes frais, ensilés ou déshydraté. Ann. Zootech., 4, 321-332.

Cordier G., 1947. De la composition de quelques produits fourragers tunisiens et de leur valeur pour l'alimentation du mouton. Ann. Serv. Bot., Agron., Tunis, 20, 25-ro8. 
Erwin E. S., Dxer I. A., Ensminger M. E., 1956. Effects of chlortetracycline, inedible animal fat and high and low quality roughage on performance of yearling steers. 2 : digestibility of dry matter, crude fiber, crude protein and ether extract. J. Anim. Sci., 15, 717-72I.

Esplin G., Hale W. H., HubBert F., TAYLor B., 1963. Effect of animal tallow and hydrolysed vegetable and animal fat on ration utilization and rumen volatile fatty acid production with fattening steers. J. Anim. Sci., 22, 695.

Fleming H. P., Etchells J. C., I967. Occurrence of an inhibitor of lactic acid bacterie in green olives. Appl. Microbiol., 15, II78-II84.

Hale W. H., Thiel E. C., Saba W. J., I965. Inflience of calcium and phosphorus levels of the ration on tallow utilization by fattening steers. Proc. West. Soc. Amer. Soc. Anim. Prod., 16, L II.

Kellner O., I924. Die Ernärhting der landwirtschaftichen Nutztiere. to Auf. Paul Parey, Berlin.

Mac Dougall E. I., I948. Studies on ruminant saliva. I. The composition and output of sheep's salive. Biochem. J., 43, 99-109.

Maymone B., Bettaglini A., Tiberio M., I962. Recherche sur la valeur nutritive du grignon d'olive Informations oléicoles internationales, 17, 65-98.

Mead S. W., Gullbert H. R., I927. In Srhneider B. H., I947. Feeds of the world. Agr. Exp. Stat. W. Virginia University, Morgantown $299 \mathrm{p}$.

Nehring K., I965. Handbuch der futtermittel, 380-382. Paul Parey, Berlin.

Peyrot E., I936. Indust. Ital. Conserv. alim., 11, $8 \mathrm{I}-82$.

Tilley J. M. A., Terry R. A., I963. A two-stage technique for the in vitro digestion of forage crops. J. Br. Grassld. Soc., 18, I04-IIr.

VAsquez-Roncero A., I965. Quimica del olivo. Grasas y aceites, 16, 292-302.

Zelter S.-Z, I 968 (Communication personnelle). 